\title{
Iron supplementation has minor effects on gut microbiota composition in overweight and obese women in early pregnancy
}

\author{
Marloes Dekker Nitert ${ }^{1,2 *}$, Luisa F. Gomez-Arango ${ }^{2}$, Helen L. Barrett ${ }^{2,3}$, H. David McIntyre ${ }^{3}$, \\ Gregory J. Anderson ${ }^{1,4}$, David M. Frazer ${ }^{4}$ and Leonie K. Callaway ${ }^{2,5}$ \\ ${ }^{1}$ School of Chemistry and Molecular Biosciences, The University of Queensland, St Lucia, QLD 4072, Australia \\ ${ }^{2} U Q$ Centre for Clinical Research, The University of Queensland, Herston, QLD 4029, Australia \\ ${ }^{3}$ Mater Health Services, Mater Hospital, South Brisbane, QLD 4001, Australia \\ ${ }^{4}$ QIMR Berghofer Medical Research Institute, Herston, QLD 4029, Australia \\ ${ }^{5}$ Obstetric Medicine, Royal Brisbane and Women's Hospital, Herston, QLD 4029, Australia \\ (Submitted 4 December 2017 - Final revision received 22 February 2018 - Accepted 23 February 2018 - First published online 23 May 2018)
}

\begin{abstract}
Fe is an essential nutrient for many bacteria, and Fe supplementation has been reported to affect the composition of the gut microbiota in both Fe-deficient and Fe-replete individuals outside pregnancy. This study examined whether the dose of Fe in pregnancy multivitamin supplements affects the overall composition of the gut microbiota in overweight and obese pregnant women in early pregnancy. Women participating in the SPRING study with a faecal sample obtained at 16 weeks' gestation were included in this substudy. For each subject, the brand of multivitamin used was recorded. Faecal microbiome composition was assessed by $16 \mathrm{~S}$ rRNA sequencing and analysed with the QIIME software suite. Dietary intake of Fe was assessed using a FFQ at 16 weeks' gestation. Women were grouped as receiving low $(<60 \mathrm{mg} / \mathrm{d}, n 94)$ or high $(\geq 60 \mathrm{mg} / \mathrm{d}$; $n$ 65) Fe supplementation. The median supplementary Fe intake in the low group was 10 (interquartile range (IQR) 5-10) $v$. 60 (IQR 60-60) mg/d in the high group $(P<0 \cdot 001)$. Dietary Fe intake did not differ between the groups $(10 \cdot 0$ (IQR 7.4-13.3) $v .9 \cdot 8$ (IQR $8 \cdot 2-13 \cdot 2) \mathrm{mg} / \mathrm{d})$. Fe supplementation did not significantly affect the composition of the faecal microbiome at any taxonomic level. Network analysis showed that the gut microbiota in the low Fe supplementation group had a higher predominance of SCFA producers. Pregnancy multivitamin Fe content has a minor effect on the overall composition of the gut microbiota of overweight and obese pregnant women at 16 weeks' gestation.
\end{abstract}

Key words: Gut microbiota: Iron: Overweight: Obesity: Pregnancy

Fe deficiency is common globally and some subgroups of the population are at particular risk $^{(1)}$. In pregnancy, circulating $\mathrm{Fe}$ levels drop owing to the increasing fetal Fe requirements as the pregnancy progresses. This often leads to Fe deficiency, which increases the risk of preterm delivery, low birth weight and neonatal mortality ${ }^{(2)}$. Fe deficiency also affects the composition of the gut microbiota and can lead to decreases in bacterial species (including Roseburia spp.) known to produce SCFA ${ }^{(3)}$, which can act as signalling molecules. Supplementation is a common strategy to reverse Fe deficiency; however, the proportion of $\mathrm{Fe}$ absorbed from oral supplements is often low ${ }^{(4)}$. Thus, non-absorbed Fe may reach the colon where it has the potential to alter the composition of the gut microbiota, as Fe is an important determinant of bacterial growth ${ }^{(5)}$.

Fe supplementation in both humans $(20 \mathrm{mg} / \mathrm{d})$ and in animal models $(10-20 \mathrm{mg} / \mathrm{kg}$ diet in rats, $0-250 \mathrm{mg} / \mathrm{kg}$ diet in piglets) has had detrimental effects on the composition of the gut microbiota $^{(3,6,7)}$. Most human supplementation studies have been performed in malnourished anaemic children in low- and middleincome countries. Fe supplementation in these populations has led to decreased abundance of Lactobacilli and increased abundance of Enterobacteriaceae and Bacteroides ${ }^{(7,8)}$. These changes have been accompanied by increases in calprotectin levels, a marker of gut inflammation ${ }^{(7)}$. Consistent with this, Fe -supplementation-associated microbiota changes are associated with increased severity of histological colitis ${ }^{(9)}$. The changes are also associated with increased frequency and severity of diarrhoeal disease $^{(6)}$, which is associated with mortality ${ }^{(10)}$.

In contrast to the findings in low- and middle-income countries, in developed countries, Fe supplementation in children and adults demonstrates different changes in the microbiota, with decreases reported in members of the Coriobacteriales and Clostridiales orders and increases in Bifidobacterium ${ }^{(11)}$. These changes do not result in diarrhoea or worsening of inflammatory bowel disease. Some gut bacteria produce phosphatidylglycerol, palmitate and palmitate derivatives, and serum levels of these are

Abbreviations: HI, high supplementary Fe intake; LI, low supplementary Fe intake; OTU, operational taxonomic units.

* Corresponding author: M. Dekker Nitert, email m.dekker@uq.edu.au 
increased in individuals receiving oral supplementary Fe compared with those supplemented intravenously ${ }^{(11)}$. A recent study in mice showed that a diet high in Fe increased the abundance of some members of the Clostriadiales and Bacteroidales orders and decreased the abundance of members of the Lactobacillales order ${ }^{(12)}$. However, the microbiota composition was differentially affected by the exact formulation of the Fe. At a consistent $\mathrm{Fe}$ dose of $50 \mathrm{mg} / \mathrm{kg}$, relative to ferrous sulphate, ferrous bisglycinate increased the abundance of members of the Bacteroidales order and decreased the abundance of Clostridiales and Bacillales orders, whereas ferric EDTA increased the abundance of some members of the Lactobacillales order but decreased the abundance of other members, as well as of members of the Clostridiales order ${ }^{(12)}$. The predicted functions of the microbiota with the different supplements also varied. Gut microbiota composition changes throughout pregnancy ${ }^{(13)}$ and has been associated with both metabolic ${ }^{(14)}$ and cardiovascular ${ }^{(15)}$ markers in the mother, as well as dietary fibre and fat intake ${ }^{(16)}$. Dietary Fe intake is positively correlated with the low-grade inflammatory marker GlycA ${ }^{(16)}$. It is possible that Fe supplementation affects maternal health by altering the composition of the maternal gut microbiota and thereby may affect fetal development. Routine Fe supplementation is not recommended for pregnant women by the Royal Australian and New Zealand College of Obstetricians and Gynaecologists. The college recommends $\mathrm{Hb}$ level measurements at the first antenatal visit and at 28 weeks' gestation and supplementation with $\geq 60 \mathrm{mg} / \mathrm{d}$ if anaemia is diagnosed. However, many pregnancy multivitamin supplements contain $\mathrm{Fe}$ at varying doses.

Given the high rates of supplementation with multivitamins in early pregnancy, this study aimed to investigate whether the dose of Fe supplementation affected the composition of the gut microbiota at baseline in a cohort of overweight and obese pregnant women participating in a randomised controlled trial of probiotics to prevent gestational diabetes mellitus (SPRING cohort).

\section{Methods}

\section{Study participants and clinical samples}

Overweight and obese pregnant women participating in the Study of Probiotics IN Gestational diabetes (SPRING, ANZCTR12611001208998) ${ }^{(17)}$ who provided a faecal sample were included in this study. All participants provided informed written consent, and the study was approved by the human research ethics committees at the Royal Brisbane and Women's Hospital (RBW/11/467) and The University of Queensland (2012000080). SPRING is a multicentre randomised clinical trial of probiotics to prevent gestational diabetes mellitus in overweight and obese women. Faecal samples were obtained at baseline (16 weeks' gestation) before randomisation and the start of the probiotics intervention. At the baseline visit, women also provided a fasting blood sample and clinical information, and completed a detailed FFQ of their nutritional intake during pregnancy (Cancer Council Victoria's Dietary Questionnaire for Epidemiological Studies DQES version 2.0 $)^{(18)}$. Use of pregnancy multivitamins and other food supplements was recorded, including brand and dose if known to the women. All brands of pregnancy multivitamins used contained ferrous Fe. Women were grouped into a low supplementary Fe intake (LI) group with 0-10 mg Fe /d ( $n$ 94) or a high supplementary Fe intake (HI) group with $\geq 60 \mathrm{mg} \mathrm{Fe} / \mathrm{d}$ ( $n$ 65). The grouping reflects the Fe content of pregnancy multivitamins available in Australia, which contain either $\leq 10 \mathrm{mg}$ of Fe or $\geq 60 \mathrm{mg}$ of Fe. The HI group includes women taking specific $\mathrm{Fe}$ supplements exceeding $60 \mathrm{mg} / \mathrm{d}(n$ 11) and those taking multivitamins containing $60 \mathrm{mg} /$ d Fe ( $n$ 54). Women whose supplementary Fe intake was uncertain were excluded from this study (see online Supplementary Fig. S1).

\section{DNA isolation}

Faecal samples were collected by the participants at home, refrigerated and stored at $-80^{\circ} \mathrm{C}$ within $24 \mathrm{~h}$ before the isolation of DNA from $250 \mathrm{mg}$ of sample using the $\mathrm{R}+\mathrm{BBC}$ method followed by the Qiagen AllPrep DNA extraction kit ${ }^{(19,20)}$. Specifically, the sample was mechanically lysed by homogenisation at $30 \mathrm{~Hz}$ for $3 \mathrm{~min}$ with $0.4 \mathrm{~g}$ of a mixture of 0.1 and $0.5 \mathrm{~mm}$ sterile zirconia beads. DNA quantity and quality was ascertained by spectrophotometry (Nanodrop ND 1000).

\section{Gut microbiota composition}

$16 S$ rRNA sequencing. The composition of the gut microbiota in faecal samples was assessed by amplification of the V6-V8 region of the $16 \mathrm{~S}$ rRNA gene using the barcoded primer set $926 \mathrm{~F}$ (5'-TCG TCG GCA GCG TCA GAT GTG TAT AAG AGA CAG AAA CTY AAA KGA ATT GRC GG-3') and 1392R (5'-GTC TCG TGG GCT CGG AGA TGT GTA TAA GAG ACA GAC GGG CGG TGW GTR C-3'). DNA isolated from Escherichia coli JM109 was used as positive control and deionised sterile water as a negative control in each PCR run. The Nextera XT V2 index kit Set A and $\mathrm{B}$ was used for barcoding the PCR products, and were purified with the AMPure XP bead system. Barcoded DNA was quantified, normalised and pooled before sequencing on the Illumina MiSeq platform at the University of Queensland's Australian Centre for Ecogenomics (ACE). The Quantitative Insights Into Microbial Ecology (QIIME) version 1.9.1 analysis pipeline $^{(21)}$ was used to join, de-multiplex and quality filter the generated forward and reverse sequences. Operational taxonomic units (OTU) were identified using the open reference OTU picking method with the Greengenes reference database using a pairwise identity threshold of $97 \%$. OTU detected in the negative controls or with an abundance of $<0.0001$ were removed from the OTU table. The OTU table was rarefied to 3000 sequences/sample before performing downstream analysis; all samples had $\geq 3000$ reads.

Statistical analysis. As the 16S rRNA data were not normally distributed, they are presented as medians and interquartile ranges. Non-parametric statistical methodology (Mann-Whitney $U$ tests, Spearman's rank correlation coefficient testing) was used and a $P$ value of $<0.05$ was considered statistically significant. The QIIME and Calypso ((http://bioinfo.qimr.edu.au/ calypso/) version $7.12^{(22)}$ software tools were used for analysis of the gut microbiota composition. Alpha diversity of the gut microbiota was determined by the Chao- 1 and Shannon indices. 
Principal component analysis was used to test for beta diversity using the Adonis statistical test for permutation multivariate ANOVA. Network analysis was performed to identify co-occurring bacteria and mutually exclusive bacteria in the LI and $\mathrm{HI}$ groups. Taxa were associated with supplementary $\mathrm{Fe}$ intake using Spearman's rho and Bray-Curtis dissimilarity distances with 1000-fold permutations. The results are reported as significant if the false discovery rate (FDR) was $<0.05$, with the degree of colouring of the nodes reflecting the level of significance of the association with supplemental Fe intake group. The predicted functions of the microbiome were analysed with the PICRUSt software tool. LefSe (linear discriminant analysis effect size) analysis was carried out to identify functional pathways discriminating between the groups.

Justification of sample size. In this analysis, sample size was determined by the availability of a faecal sample and information about supplemental Fe intake. However, the group sizes of ninety-four and sixty-five individuals, respectively, enable to distinguish a $30 \%$ change in abundance between the groups for bacteria with a relatively low mean abundance of $1 \%$ of the gut microbiota composition and a SD of $0.8 \%$.

\section{Results}

\section{Participants}

Of the 210 participants in the SPRING study with faecal samples, 159 women had detailed supplementary Fe intake information, with ninety-four women having low supplementary Fe intake (LI group) and sixty-five women having high supplementary Fe intake (HI group) (Table 1). The Fe in the supplements taken by the women in the LI group consisted of either ferrous sulphate or ferrous glycinate. In the HI group all the supplemental Fe was as ferrous sulphate. The groups did not differ in maternal age, BMI, gestational age at study entry, systolic or diastolic blood pressure or fasting glucose levels. In addition, dietary Fe intake levels were similar in both groups, about $10 \mathrm{mg} / \mathrm{d}$. Dietary protein intake levels were also similar in both groups and correlated strongly with dietary Fe intake levels $(\rho=0 \cdot 88, P<0 \cdot 0001)$.

\section{Overall gut microbiota composition}

Alpha diversity was not different between the groups (results not shown). Beta diversity was significantly different between the groups ( $R=0.037, P=0.031$ ) (Fig. $1(\mathrm{a})$ ); however, the $R$-value is close to 0 , indicating that the difference was small. Furthermore, there were no major clustering differences in gut microbial composition between the groups at any taxonomic level (genus level presented as Fig. 1(b)). The abundance of bacteria previously noted in the literature to be differentially affected by supplementary Fe intake was analysed (Table 2). The abundance of the genera Roseburia and Ruminococcus was significantly lower in the HI group ( $P=0.045$ and $P=0.038)$, but this was no longer significant after correction for multiple testing (FDR $0 \cdot 23$ and $0 \cdot 18$, respectively). Network analyses showed that the gut microbiota of women with low supplementary Fe intake was dominated at the genus level by bacteria known to contain shortchain fatty acid producers, including Ruminococcus, Suterella and Lachnospira, but also the lactate producer Lactobacillus (Fig. 2). In women with high supplementary Fe intake, only Acidaminococcus was over-represented. However, it should be noted that the differences are small, as indicated by the lack of bright nodes in the network.

\section{Differences in the predicted function of the gut microbiota}

The predicted functions of the gut microbiota in the LI and HI groups were compared. The gut microbiota in LI group was associated with more pronounced butanoate metabolism and lipid biosynthesis proteins, whereas in the HI group amino acid metabolism and siderophore biosynthesis were more pronounced (Fig. 3(a) and (b)).

\section{Discussion}

The results of this study show that the routine supplementation with $\mathrm{Fe}$ in multivitamin capsules in early pregnancy is not

Table 1. Participant characteristics

(Medians and interquartile ranges (IQR) and ranges)

\begin{tabular}{|c|c|c|c|c|c|}
\hline & \multicolumn{2}{|c|}{ LI group } & \multicolumn{2}{|c|}{$\mathrm{HI}$ group } & \multirow[b]{2}{*}{$P$} \\
\hline & Median & IQR & Median & IQR & \\
\hline$n$ & \multicolumn{2}{|c|}{94} & \multicolumn{2}{|c|}{65} & \\
\hline Maternal age (years) & 32 & $29-34$ & 32 & $27-35$ & 0.79 \\
\hline $\mathrm{BMI}\left(\mathrm{kg} / \mathrm{m}^{2}\right)$ & $31 \cdot 6$ & $28 \cdot 2-36 \cdot 1$ & $31 \cdot 3$ & $28 \cdot 3-37 \cdot 5$ & 0.40 \\
\hline Parity & 1 & $0-2$ & 1 & $0-1$ & 0.08 \\
\hline Gestational age (d) & 108 & $101-112$ & 109 & $101-113$ & 0.79 \\
\hline Systolic BP (mmHg) & 110 & $104-116$ & 110 & $105-116$ & 0.79 \\
\hline Diastolic BP (mmHg) & 70 & $62-74$ & 70 & $60-75$ & 0.76 \\
\hline Fasting glucose (mmol/l) & $5 \cdot 0$ & $4 \cdot 6-5 \cdot 2$ & 4.8 & $4 \cdot 6-5 \cdot 2$ & 0.34 \\
\hline Supplementary Fe intake $(\mathrm{mg} / \mathrm{d})$ & & & & & $<0.001$ \\
\hline Median & \multirow{2}{*}{\multicolumn{2}{|c|}{$\begin{array}{c}10 \\
0-10\end{array}$}} & \multirow{2}{*}{\multicolumn{2}{|c|}{$\begin{array}{c}60 \\
60-115\end{array}$}} & \\
\hline Range & & & & & \\
\hline Dietary Fe intake (mg/d) & $10 \cdot 0$ & $7 \cdot 4-13 \cdot 3$ & $9 \cdot 8$ & $8 \cdot 2-13 \cdot 2$ & 0.86 \\
\hline Dietary protein intake $(\mathrm{g} / \mathrm{d})$ & 74 & $58-94$ & 75 & $62-96$ & 0.42 \\
\hline Infant birth weight $(\mathrm{g})$ & 3655 & $3223-3951$ & 3565 & 3179-3892 & 0.37 \\
\hline
\end{tabular}

LI, low supplementary Fe intake; HI, high supplementary Fe intake; BP, blood pressure. 
(a)

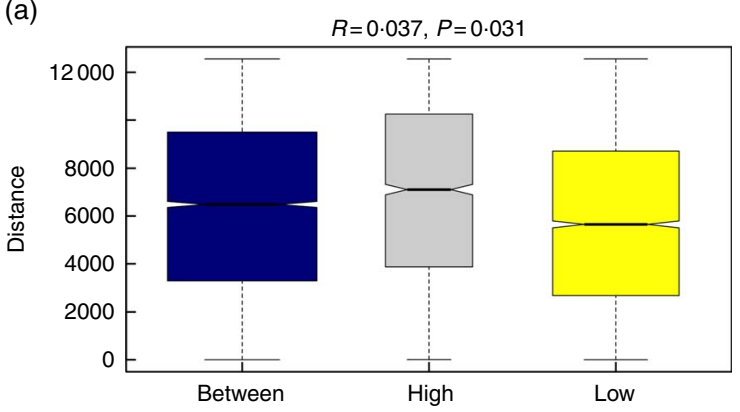

(b)

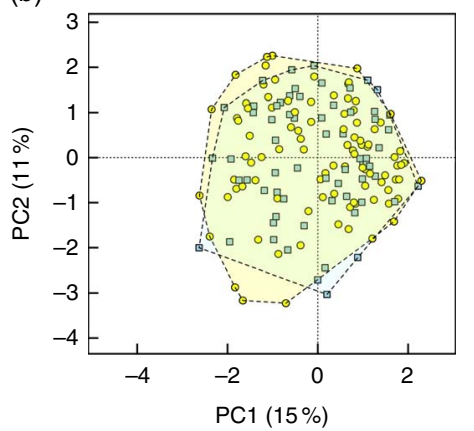

Fig. 1. Overall composition of the gut microbiota in women with high or low supplementary iron intake. (a) Beta-diversity plot for supplementary iron intake showing diversity of the gut microbiota within the high-iron supplementation group, within the low supplementation group and between the groups. Beta-diversity was assessed by Anosim and was found to be significantly different between the groups. (b) Principal component analysis (PCA) plot at the genus level for women with high ( $\square \square$ ) and low $(O)$ supplementary iron intake.

Table 2. Analysis of the abundance of bacteria previously shown to be affected by supplemental iron intake

\begin{tabular}{lccll}
\hline & $\begin{array}{c}\text { LI group (median } \\
\text { abundance) }\end{array}$ & $\begin{array}{c}\text { HI group (median } \\
\text { abundance) }\end{array}$ & $P$ & $\begin{array}{l}\text { FDR } \\
\text { value }\end{array}$ \\
\hline Enterococcus & $\mathrm{N} / \mathrm{A}$ & $\mathrm{N} / \mathrm{A}$ & & \\
Enterobacteriaceae & 5.14 & 5.38 & 0.31 & 1 \\
Lactobacillus & 0 & 0 & 0.56 & 0.92 \\
Bacteroides & 12.91 & 12.63 & 0.53 & 0.92 \\
Roseburia & 6.74 & 5.71 & 0.045 & 0.23 \\
Clostridium & 7.28 & 7.36 & 0.76 & 0.92 \\
Eubacterium & 0 & 0 & 0.42 & 0.92 \\
Ruminococcus & 10.32 & 9.91 & 0.038 & 0.18 \\
\hline
\end{tabular}

$\mathrm{LI}$, low supplementary $\mathrm{Fe}$ intake; $\mathrm{HI}$, high supplementary $\mathrm{Fe}$ intake; FDR, false discovery rate.

associated with under- or over-abundance of specific genera in the gut microbiota. In the network analysis, there was a slightly higher predominance of bacteria that include known shortchain fatty acid producers in the women who had no/low levels of Fe supplementation. To our knowledge, this is the first study of the effects of Fe supplementation on the composition of the gut microbiota in pregnancy.

Functional predictions of the gut microbiota in women with low supplementary Fe indicated that butanoate (butyrate) metabolism was increased. Higher faecal butyrate concentrations have been associated with increased abundance of the genus Roseburia from the family Lachnospiraceae ${ }^{(23)}$. In our study, Roseburia abundance tended to be higher in the gut microbiota of women with low Fe supplementation. These findings are consistent with rodent studies that reported that Fe supplementation reduced the abundance of Roseburia spp. in Fe-deficient animals ${ }^{(9)}$. In humans, Fe supplementation has also been reported to decrease the abundance of Roseburia ${ }^{(12)}$. Similarly, the genus Ruminococcus in the family Ruminococcaceae is also a known butyrate producer and trended towards lower abundance in the gut microbiota of women with high Fe supplementation $^{(24)}$. In the gut, increased butyrate metabolism may contribute to a stronger intestinal barrier, a thicker mucus layer and reduction of pro-inflammatory cytokines ${ }^{(25)}$. Given the changes noted with Fe supplementation, the decreases in SCFA producers, which mainly synthesise acetate, propionate and butyrate, may alter gut barrier function. Butyrate metabolism is decreased in the gut microbiota of patients with known gut barrier function disorders such as Crohn's disease ${ }^{(26)}$. Decreased butyrate metabolism has previously been described in the placental microbiota of women with excess gestational weight gain who delivered prematurely ${ }^{(27)}$. In pregnant women, impaired intestinal barrier function could increase the inflammatory state of the mother, which could have an impact on the size of the baby. However, there was no difference in infant birth size in our cohort. Our findings with Fe supplementation need to be investigated further to explore the relationships between Fe supplementation and other obstetric outcomes.

The network analysis in women with higher supplemental Fe intake showed a microbiota with a higher abundance of Acidaminococcus. Acidaminococcus, a member of the Firmicutes phylum, has been associated with changes in glucose metabolism and obesity. In patients undergoing bariatric surgery, the abundance of Acidaminococcus increases; however, this was not correlated with any metabolic markers including fasting serum glucose, insulin or cholesterol levels in the patients ${ }^{(28)}$. Acidaminococcus spp. are known amino-acidfermenting bacteria, and exclusively metabolise glutamate for carbon and energy supply. It has been hypothesised that the increase in undigested protein after bariatric surgery could potentially explain the increased abundance of Acidaminococcus $^{(28)}$. However, in our cohort, dietary protein intake was similar between the groups, and it is likely that, in healthy pregnant women, the majority of the protein is digested before entering the colon. Undigested protein is therefore unlikely to explain the difference in Acidaminococcus in our study.

Low protein intake and competition for glutamate between the host gut epithelium and Acidaminococcus may negatively affect gut barrier function and growth in children, potentially by affecting nitrogen metabolism and urea recycling in the gut lumen ${ }^{(29)}$. In undernourished children, Acidaminococcus abundance is higher in children with stunting and negatively correlated with height-over-age $z$ scores $^{(29)}$. Acidaminococcus can be detected in children with newly diagnosed type 1 diabetes, but not in their matched normoglycaemic counterparts ${ }^{(30)}$. Type 1 diabetes has also been associated with increased gut leakiness ${ }^{(31)}$, providing a common mechanism for the gut microbiota to affect the health of the host. 


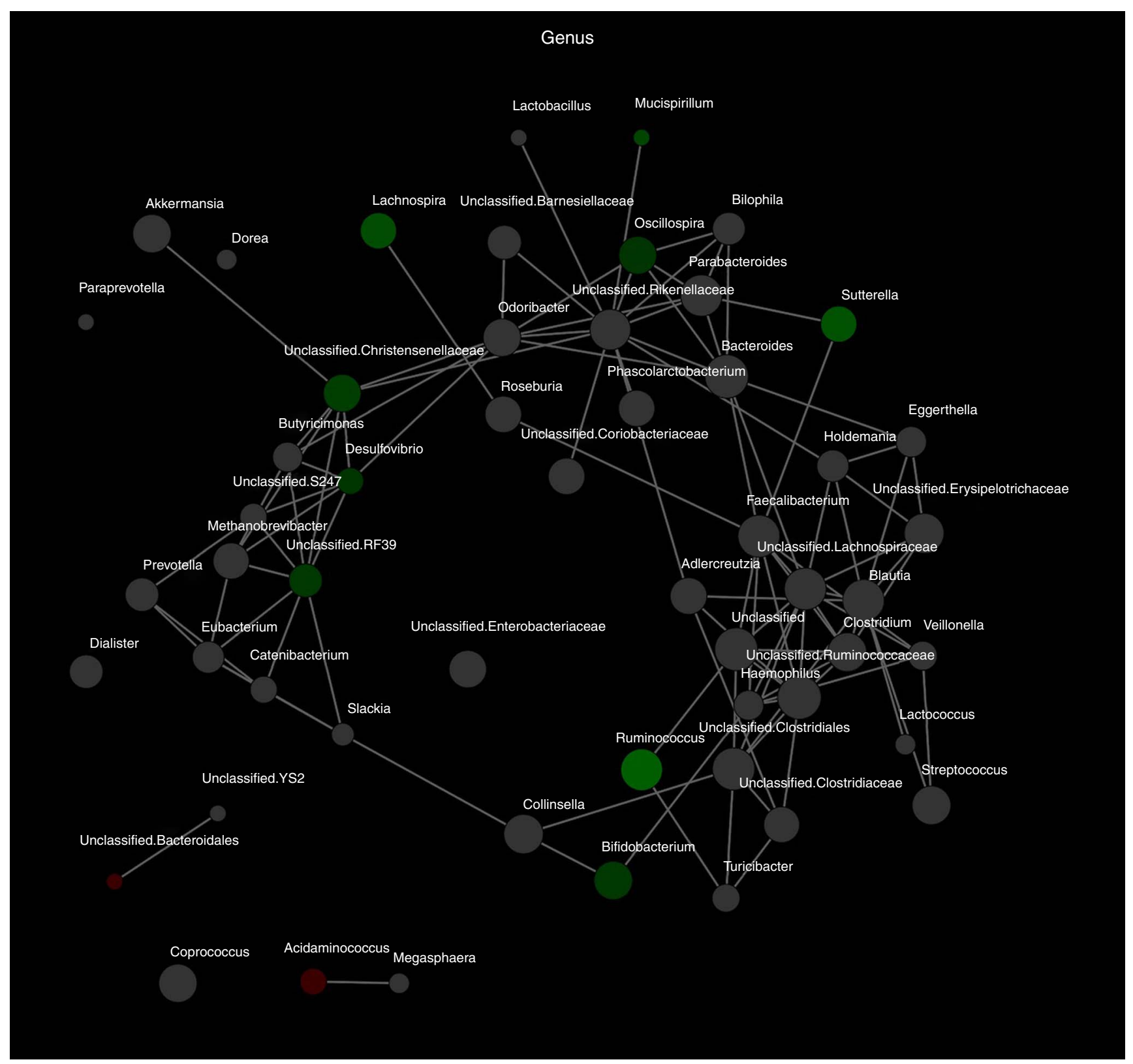

Fig. 2. Differences in microbiota composition according to high and low supplementary iron intake. Network analysis based on high ( $n 65)$ and low $(n 94)$ supplementary iron intake. Genera are represented as nodes. Green nodes indicate genera over-represented in the low-iron group. Red nodes indicate genera over-represented in the high-iron group. Colour intensity explains the strength of the associations with supplementary iron intake, with more colour representing a stronger association.

In our study, the microbiota of women receiving high $\mathrm{Fe}$ supplementation had higher predicted siderophore biosynthesis and amino acid metabolism. This was an unexpected finding. Siderophores are low-molecular-weight Fe carriers and serve to bind $\mathrm{Fe}$, especially $\mathrm{Fe}^{3+}$, with high affinity ${ }^{(32)}$. They are usually produced by bacteria and fungi in response to low-Fe environments ${ }^{(32)}$, which is opposite to our HI group. Siderophores comprise multiple different compounds, which can have distinctive functions and affinities for ferric and ferrous $\mathrm{Fe}$, and bacteria may use a composite of different siderophores to ensure survival $^{(33)}$. Our predictive functional analysis does not distinguish between different forms of siderophores. The predominance of siderophore biosynthesis in the HI group may reflect a microbiota where bacteria capable of producing siderophores are over-represented, as some bacteria, including certain Lactobacillus spp., do not synthesise siderophores ${ }^{(32)}$. Most supplements contain ferrous $\mathrm{Fe}\left(\mathrm{Fe}^{2+}\right)$, which has been shown to down-regulate the synthesis of siderophores in Fe -replete states, possibly to prevent oxidative stress ${ }^{(34)}$. It is possible that, although the microbiota of women receiving high Fe supplementation contains more bacterial species that produce siderophores, the actual rates of synthesis are unchanged. We do not know the Fe status or the duration of Fe supplement use in our cohort, and thus it is possible that women with a history or presence of Fe deficiency had chosen to commence the higher-Fe -containing Fe supplement. The 
(a)
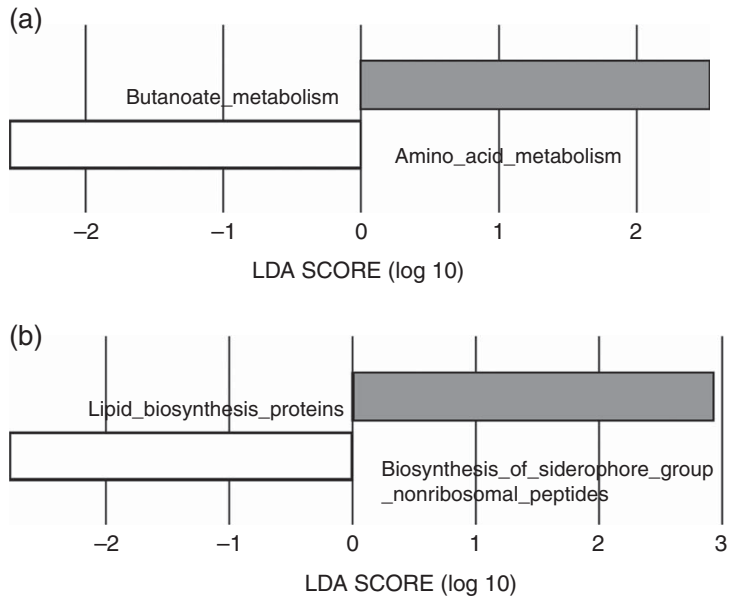

Fig. 3. Predictive functional analysis of metabolism and biosynthesis of the gut microbiota in the low-iron (LI) and high-iron ( $\mathrm{HI}$ ) supplementation groups. (a) Metabolic pathways significantly different in the $\mathrm{LI}(\square)$ and $\mathrm{HI}(\square)$ groups. (b) Significantly different predicted biosynthetic pathways in the $\mathrm{LI}$ and $\mathrm{HI}$ groups. LDA score, linear discriminant analysis score of predicted pathways discriminating between $\mathrm{LI}$ and $\mathrm{HI}$ groups.

conditions and bacteria required for actual bacterial siderophore production in the human intestine are still largely unknown ${ }^{(33)}$, and our findings need to be confirmed in other data sets.

In this cohort of women, we were not able to definitively determine their Fe status. However, although the HI group may include some women with low Fe status who were prescribed Fe supplementation by their health care provider, the majority of this group (83\%) used a multivitamin-containing $60 \mathrm{mg} /$ capsule of Fe as indicated by the median and range displayed in Table 1 . It is likely that the study population resembles the general obstetric population in Fe status and multivitamin use in Australia $^{(35)}$, where up to $97.5 \%$ use multivitamins throughout the first trimester. All multivitamin supplements used by the women in this study contained ferrous $\mathrm{Fe}$, with the high-Fe supplement consisting solely of ferrous sulphate, and the women in the LI group were taking a supplement containing either ferrous sulphate or ferrous glycinate. There was no indication that the form of Fe altered the gut microbiota. The fact that there were no specific genera of bacteria that were altered in women with high Fe supplementation suggests that this level of supplementation does not markedly alter the composition and thereby the function of the gut microbiota. Changes to the gut microbiota in response to supplementation or dietary changes are generally rapid ${ }^{(36)}$, and it is therefore unlikely that the lack of differences observed is due to the duration of supplementation. Given that this cohort only included overweight and obese women, the impact of multivitamin supplementation incorporating Fe on the gut microbiota in lean women needs to be examined. However, the fact that there were no differences in BMI between the groups in this study suggests that our findings are independent of BMI. Furthermore, whether or not maternal Fe status is associated with changes in the gut microbiota of pregnant women and whether Fe supplementation rectifies any alterations in women with low Fe status remain to be determined. Furthermore, recent studies emphasised the effects of dietary fibre, fat and vitamin intake on gut microbiota composition in early pregnancy and early postpartum $^{(16,37)}$. It is possible that the differences in dietary fibre and fat intake between individual women mask any differences due to supplementary Fe intake. However, this again suggests that supplementary Fe intake only has a minor effect on gut microbiota composition. Last, this study was powered to observe a difference of $\geq 30 \%$ in abundance of relatively lowabundant bacteria (mean 1 (SD 0.8)\%). The network analyses suggest that smaller changes may be present: if the functional outcome of the small changes is in the same direction, these may be physiologically important. A larger $16 \mathrm{~S}$ rRNA study or a meta-genomic analysis of the genes present or expressed by the bacteria could clarify this further.

\section{Conclusion}

In conclusion, the results of this study suggest that the Fe levels up to $60 \mathrm{mg} / \mathrm{d}$ in pregnancy multivitamins do not significantly alter the overall composition of the gut microbiota in early pregnancy. Network analyses indicated that supplementation with lower amounts of Fe is correlated with a slightly higher abundance of SCFA producers, which may be beneficial in maintaining gut barrier integrity.

\section{Acknowledgements}

The authors thank the SPRING participants; the SPRING trial group included Katie Foxcroft, Anne Tremellen, Sharney Grant, Dr Jacinta Tobin, Dr Shelley Wilkinson, Dr Chris McSweeney, Prof Peter O'Rourke and Dr Barbara Lingwood.

L. F. G.-A. is supported by a Colciencias scholarship from the government of Colombia. H. L. B. is supported by an National Health \& Medical Research Council (NHMRC) Early Career Fellowship (grant no. APP1120070). The SPRING study is supported by the NHMRC (grant no. 1028575), Diabetes Australia Research Programme and the Royal Brisbane and Women's Hospital Foundation.

M. D. N. designed the research, conducted the study and analysed the data, and wrote and edited the manuscript. L. F. G.-A. researched data and performed analysis. L. F. G.-A., H. L. B., D. M. F., H. D. M., L. K. C. and G. J. A. provided intellectual input and edited the manuscript. H. L. B. and D. M. F. designed the research. H. D. M. and L. K. C. provided samples.

The SPRING study has received probiotics and placebo products from Chr Hansen A/S. The authors declare that there are no conflicts of interest.

\section{Supplementary material}

For supplementary material/s referred to in this article, please visit https://doi.org/10.1017/S0007114518001149

\section{References}

1. Zimmermann MB \& Hurrell RF (2007) Nutritional iron deficiency. Lancet 370, 511-520.

2. Coad J \& Conlon C (2011) Iron deficiency in women: assessment, causes and consequences. Current Opin Clin Nutr Metab Care 14, 625-634. 
3. Dostal A, Chassard C, Hilty FM, et al. (2012) Iron depletion and repletion with ferrous sulfate or electrolytic iron modifies the composition and metabolic activity of the gut microbiota in rats. J Nutr 142, 271-277.

4. Cook JD \& Reddy MB (1995) Efficacy of weekly compared with daily iron supplementation. Am J Clin Nutr 62, 117-120.

5. Prentice AM, Mendoza YA, Pereira D, et al. (2017) Dietary strategies for improving iron status: balancing safety and efficacy. Nutr Rev 75, 49-60.

6. Lee SH, Shinde P, Choi J, et al. (2008) Effects of dietary iron levels on growth performance, hematological status, liver mineral concentration, fecal microflora, and diarrhea incidence in weanling pigs. Biol Trace Elem Res 126, 57-68.

7. Zimmermann MB, Chassard C, Rohner F, et al. (2010) The effects of iron fortification on the gut microbiota in African children: a randomized controlled trial in Cote d'Ivoire. Am J Clin Nutr 92, 1406-1415.

8. Krebs NF, Sherlock LG, Westcott J, et al. (2013) Effects of different complementary feeding regimens on iron status and enteric microbiota in breastfed infants. J Pediatr 163, 416-423. e414.

9. Dostal A, Lacroix C, Pham VT, et al. (2014) Iron supplementation promotes gut microbiota metabolic activity but not colitis markers in human gut microbiota-associated rats. Br J Nutr 111, 2135-2145.

10. Kotloff KL (2017) The burden and etiology of diarrheal illness in developing countries. Pediatr Clin North Am 64, 799-814.

11. Lee T, Clavel T, Smirnov K, et al. (2017) Oral versus intravenous iron replacement therapy distinctly alters the gut microbiota and metabolome in patients with IBD. Gut 66, 863.

12. Constante M, Fragoso G, Lupien-Meilleur J, et al. (2017) Iron supplements modulate colon microbiota composition and potentiate the protective effects of probiotics in dextran sodium sulfate-induced colitis. Inflamm Bowel Dis $\mathbf{2 3}$, 753-766.

13. Koren O, Goodrich JK, Cullender TC, et al. (2012) Host remodeling of the gut microbiome and metabolic changes during pregnancy. Cell 150, 470-480.

14. Gomez-Arango LF, Barrett HL, McIntyre HD, et al. (2016) Connections between the gut microbiome and metabolic hormones in early pregnancy in overweight and obese women. Diabetes 65, 2214-2223.

15. Gomez-Arango LF, Barrett HL, McIntyre HD, et al. (2016) Increased systolic and diastolic blood pressure is associated with altered gut microbiota composition and butyrate production in early pregnancy. Hypertension 68, 974-981.

16. Röytiö H, Mokkala K, Vahlberg T, et al. (2017) Dietary intake of fat and fibre according to reference values relates to higher gut microbiota richness in overweight pregnant women. $\mathrm{BrJ}$ Nutr 118, 343-352.

17. Nitert MD, Barrett H, Foxcroft K, et al. (2013) SPRING: an RCT study of probiotics in the prevention of gestational diabetes mellitus in overweight and obese women. BMC Pregnancy Childbirth 13, 50.

18. Giles GG IP (1996) Dietary Questionnaire for Epidemiological Studies (Version 2). Melbourne: The Cancer Council Victoria.

19. Gomez-Arango LF, Barrett HL, McIntyre HD, et al. (2016) Connections between the gut microbiome and metabolic hormones in early pregnancy in overweight and obese women. Diabetes 65, 2214-2223.
20. Yu Z \& Morrison M (2004) Improved extraction of PCR-quality community DNA from digesta and fecal samples. BioTechniques 36, 808-812.

21. Caporaso JG, Kuczynski J, Stombaugh J, et al. (2010) QIIME allows analysis of high-throughput community sequencing data. Nat Methods 7, 335-336.

22. Zakrzewski M, Proietti C, Ellis JJ, et al. (2016) Calypso: a userfriendly web-server for mining and visualizing microbiomeenvironment interactions. Bioinformatics 33, 782-783.

23. Barton W, Penney NC, Cronin O, et al. (2017) The microbiome of professional athletes differs from that of more sedentary subjects in composition and particularly at the functional metabolic level. Gut 67, 625-633.

24. Zhang LS \& Davies SS (2016) Microbial metabolism of dietary components to bioactive metabolites: opportunities for new therapeutic interventions. Genome Med 8, 46.

25. van der Beek CM, Dejong CHC, Troost FJ, et al. (2017) Role of short-chain fatty acids in colonic inflammation, carcinogenesis, and mucosal protection and healing. Nutr Rev 75, 286-305.

26. Wright EK, Kamm MA, Teo SM, et al. (2015) Recent advances in characterizing the gastrointestinal microbiome in Crohn's disease: a systematic review. Inflamm Bowel Dis 21, 1219-1228.

27. Antony KM, Ma J, Mitchell KB, et al. (2015) The preterm placental microbiome varies in association with excess maternal gestational weight gain. Am J Obstet Gynecol 212, 653.e651-653.e616.

28. Patrone V, Vajana E, Minuti A, et al. (2016) Postoperative changes in fecal bacterial communities and fermentation products in obese patients undergoing bilio-intestinal bypass. Front Microbiol 7, 200.

29. Gough EK, Stephens DA, Moodie EEM, et al. (2015) Linear growth faltering in infants is associated with Acidaminococcus sp. and community-level changes in the gut microbiota. Microbiome 3, 24.

30. Qi C-J, Zhang Q, Yu M, et al. (2016) Imbalance of fecal microbiota at newly diagnosed type 1 diabetes in Chinese children. Chin Med J (Engl) 129, 1298-1304.

31. Li X \& Atkinson MA (2015) The role for gut permeability in the pathogenesis of type 1 diabetes - a solid or leaky concept? Pediatr Diabetes 16, 485-492.

32. Neilands JB (1995) Siderophores: structure and function of microbial iron transport compounds. J Biol Chem 270, 26723-26726.

33. Ellermann M \& Arthur JC (2017) Siderophore-mediated iron acquisition and modulation of host-bacterial interactions. Free Radic Biol Med 105, 68-78.

34. Touati D, Jacques M, Tardat B, et al. (1995) Lethal oxidative damage and mutagenesis are generated by iron in delta fur mutants of Escherichia coli: protective role of superoxide dismutase. J Bacteriol 177, 2305-2314.

35. Shand AW, Walls M, Chatterjee R, et al. (2016) Dietary vitamin, mineral and herbal supplement use: a cross-sectional survey of before and during pregnancy use in Sydney, Australia. Aust $N Z$ J Obstet Gynaecol 56, 154-161.

36. David LA, Maurice CF, Carmody RN, et al. (2013) Diet rapidly and reproducibly alters the human gut microbiome. Nature $\mathbf{5 0 5}, 559$.

37. Mandal S, Godfrey KM, McDonald D, et al. (2016) Fat and vitamin intakes during pregnancy have stronger relations with a pro-inflammatory maternal microbiota than does carbohydrate intake. Microbiome $4,55$. 